\title{
Blocking NUPR1 protein, a successful approach for pancreatic adenocarcinoma treatment
}

Jose L. Neira, Jennifer Bintz, Maria Arruebo, Bruno Rizzuti, Thomas Bonacci, Sonia Vega, Angel Lanas, Adrian Velazquez-Campoy, Juan L. Iovanna, Olga Abian

Background: Pancreatic ductal adenocarcinoma (PDAC) is the most common type of pancreatic malignancy. Nuclear protein 1 (NUPR1) is a recognized protein, over-expressed and involved in PDAC development. NUPR1 belongs to the special class of intrinsically disordered proteins (IDPs) and it is implicated in cell signaling and regulatory functions. The multifunctional nature of NUPR1 renders it as an attractive target for drug design and development. Aim: Identify a small molecule inhibiting protein-protein interactions in NUPR1 and able to interfere with any of NUPR1 key oncogenic activities, thus, constituting a new chemotherapy strategy against PDAC. Methods: Ligand-induced stabilization against thermal denaturation (thermal-shift assay) was employed for identifying potential NUPR1-interacting compounds. An in vitro molecular screening based on thermal denaturation of NUPR1 in the presence of a variety of potential ligands was performed using a collection of 1120 compounds. All compounds are FDA-approved drugs for different therapeutic indications, exhibiting high chemical and pharmacological diversity, as well as good bioavailability and safety parameters in humans. Direct interaction of selected compounds with NUPR1 was assessed experimentally (calorimetry, fluorescence spectroscopy, nuclear magnetic resonance, and proximity ligation assay) and computationally (molecular dynamics simulations). Compound efficacy was determined in PDAC-derived cell-based assays and in vivo assays on xenografted PDAC-derived cells in mice. Comparisons of treatment outcome were tested for statistical significance by using the t-test, and statistical significance was assumed at a p-value lower than 0.05. Results: Fifteen candidates were selected, and their interactions with NUPR1 were characterized. In vitro experiments with MiaPaCa-2 cells treated using $10 \mu \mathrm{M}$ of compounds for 6 days showed that two of the compounds ( $\mathrm{C} 13$ and $\mathrm{C} 15$ ) were very efficient in diminishing cell viability (10 \pm $3 \%$ and $26 \pm 7 \%$, respectively; assays in triplicates ( $p \leq 0.01$ ). These values were similar to those obtained with oxaliplatin ( $10 \pm 2 \%$; $p \leq 0.01$ ). Also, they reduced cell migration (from $10-20 \%$ wound-healing ability compared to $50 \%$ in control assays; $p \leq 0.05$ ) and colony formation (completely suppressed in the presence of both compounds; $p \leq 0.01$ ). In addition, the most promising compound, C15, interfered with the interaction of NUPR1 with MSL1, one of the NUPR1 binding partners (left figures). The administration of a $10 \mathrm{mg} / \mathrm{Kg}$ dose of C15 promoted complete arrest of tumor development on xenografted PDAC-derived cells in mice (right figures). Conclusion: We report the discovery of a compound specifically active against PDAC and interfering with NUPR1. In addition, we demonstrate that it is possible to identify small molecules able to modulate the function of complex targets such as IDPs.
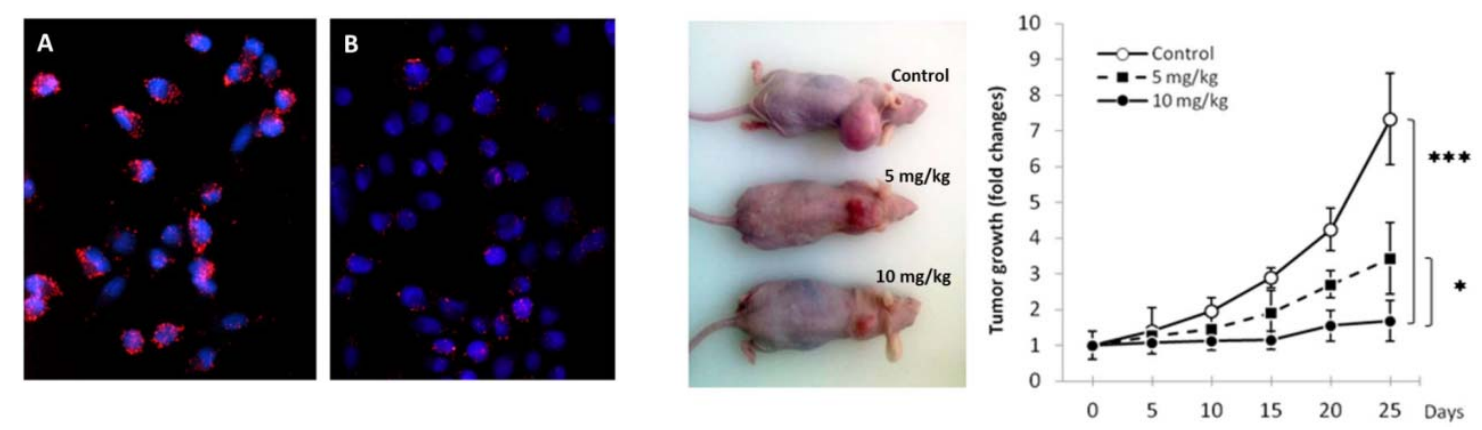